\title{
Molecular expression, characterization and mechanism of ALAS2 gain-of-function mutants
}

\author{
Vassili Tchaikovskii, Robert J. Desnick and David F. Bishop* (1)
}

\begin{abstract}
Background: X-linked protoporphyria (XLP) (MIM 300752) is an erythropoietic porphyria due to gain-of-function mutations in the last exon (Ducamp et al., Hum Mol Genet 22:1280-88, 2013) of the erythroid-specific aminolevulinate synthase gene (ALAS2). Five ALAS2 exon 11 variants identified by the NHBLI Exome sequencing project (p.R559H, p.E565D, p.R572C, p.S573F and p.Y586F) were expressed, purified and characterized in order to assess their possible contribution to XLP. To further characterize the XLP gain-of-function region, five novel ALAS2 truncation mutations (p.P561X, p.V562X, p.H563X, p.E569X and p.F575X) were also expressed and studied.
\end{abstract}

Methods: Site-directed mutagenesis was used to generate ALAS2 mutant clones and all were prokaryotically expressed, purified to near homogeneity and characterized by protein and enzyme kinetic assays. Standard deviations were calculated for 3 or more assay replicates.

Results: The five ALAS2 single nucleotide variants had from 1.3- to 1.9-fold increases in succinyl-CoA $V_{\max }$ and 2- to 3 -fold increases in thermostability suggesting that most could be gain-of-function modifiers of porphyria instead of causes. One SNP (p.R559H) had markedly low purification yield indicating enzyme instability as the likely cause for XLSA in an elderly patient with $x$-linked sideroblastic anemia. The five novel ALAS2 truncation mutations had increased $V_{\max }$ values for both succinyl-CoA and glycine substrates (1.4 to 5.6-fold over wild-type), while the $K_{m} s$ for both substrates were only modestly changed. Of interest, the thermostabilities of the truncated ALAS2 mutants were significantly lower than wild-type, with an inverse relationship to $V_{\max }$ fold-increase.

Conclusions: Patients with porphyrias should always be assessed for the presence of the ALAS2 gain-of-function modifier variants identified here. A key region of the ALAS2 carboxyterminal region is identified by the truncation mutations studied here and the correlation of increased thermolability with activity suggests that increased molecular flexibility/active site openness is the mechanism of enhanced function of mutations in this region providing further insights into the role of the carboxyl-terminal region of ALAS2 in the regulation of erythroid heme synthesis.

Keywords: X-linked sideroblastic anemia, Porphyria, Erythropoietic, Polymorphism, Single nucleotide, Glycine; succinylcoenzyme a

\footnotetext{
* Correspondence: dfbishop@earthlink.net

Department of Genetics and Genomic Sciences, Icahn School of Medicine at

Mount Sinai New York, New York 10029, USA
}

(c) The Author(s). 2019 Open Access This article is distributed under the terms of the Creative Commons Attribution 4.0 International License (http://creativecommons.org/licenses/by/4.0/), which permits unrestricted use, distribution, and reproduction in any medium, provided you give appropriate credit to the original author(s) and the source, provide a link to the Creative Commons license, and indicate if changes were made. The Creative Commons Public Domain Dedication waiver (http://creativecommons.org/publicdomain/zero/1.0/) applies to the data made available in this article, unless otherwise stated. 


\section{Introduction}

Erythroid-specific 5-aminolevulinate synthase (ALAS2, EC 2.3.1.37), is the first and rate-limiting enzyme in the erythroid heme biosynthetic pathway It is encoded by an X-linked gene (Bishop et al. 1990) and is expressed in fetal liver and adult bone marrow to provide sufficient heme for hemoglobin synthesis. ALAS2 uses pyridoxal-5' phosphate to catalyze the condensation of glycine and succinyl-CoA to produce 5-aminolevulinic acid (ALA), the first heme precursor. X-Linked Sideroblastic Anemia (XLSA) is caused by over 80 mutations in ALAS2 (Cotter et al. 1992; Stenson et al. 2017), with nearly all located in exons 5 through 11 . Recently, attention has been focused on mutations in the 33-residue carboxyl-terminal exon 11, since these mutations can cause XLSA without decreasing the in vitro catalytic activity (Bishop et al. 2012) or, more typically, cause X-Linked Protoporphyria (XLP) via overproduction of ALA (Whatley et al. 2008) and subsequent heme biosynthetic intermediates without causing anemia. These XLP gain-of-function mutations result in extreme photosensitivity due to protoporphyrin IX accumulation and the development of severe phototoxic reactions upon exposure to sunlight. The divergent and unique consequences of ALAS2 exon 11 mutations suggested that this exon serves as an intrinsic regulator of ALA synthesis (Kadirvel et al. 2012; Fratz et al. 2015). This 3' carboxyl-terminal extension encodes a well-conserved amino acid region in higher eukaryotes, but is not present in bacteria, the source of the first crystal structure of ALAS (Astner et al. 2005). Recently, the yeast ALAS 3D structure was determined providing the first insights into the location of the carboxyl-terminal region partially homologous to human exon 11 (Brown et al. 2018).

Previously, we demonstrated that purified recombinant human ALAS2 enzymes from XLP patients with mutations in the gain-of-function region led to 1.6- to 3.1-fold increased enzymatic specific activity (Bishop et al. 2013). With others, we established the gain-of-function region to be between ALAS2 residues 533-580 (Bishop et al. 2013; Ducamp et al. 2013).

To assess whether other known exon 11 missense mutations (identified as polymorphisms in the NHLBI Exome Sequencing Project [ESP] Exome Variant Server database of 13,006 chromosomes [release ESP6500]) (Exome Variant Server 2017) may cause XLSA or XLP, five novel missense mutations were identified as being the only significant enzyme variants in ALAS2 exon 11. These variants were introduced into human ALAS2 expression constructs, overexpressed, purified, and characterized. To further characterize this region, five truncation mutations were also generated and characterized to help fine-map the region of greatest gain-of-function.

\section{Materials and methods}

The reagents used, preparation of ALAS2 expression constructs, and the expression and purification of ALAS2 recombinant enzymes were as previously described (Bishop et al. 2013). Positive clones for missense mutations (p.R559H, p.E565D, p.R572C, p.S573F, \& p.Y586F) were identified by restriction analysis and confirmed by subsequent sequence analyses. Truncated constructs (p.P561X, p.V562X, p.H563X, p.E569X and p.F575X) were made by deleting appropriate coding nucleotides of ALAS2 exon 11 using primers that included stop codons TAG or TAA as shown in Additional file 1: Table S1.

Enzyme protein concentrations, kinetics, and thermostability studies were conducted using endpoint assays as previously described (Bishop et al. 2013). Note that our choice of $45^{\circ} \mathrm{C}$ as the denaturation temperature for stability studies was chosen to maximize discrimination between structural forms. Oligonucleotide primers used for mutagenesis and sequence analyses are listed in Additional file 1 Table S1.

\section{Results \\ Expression and purification of wild-type and mutant ALAS2 enzymes}

Each of the five human ALAS2 missense mutations (p.R559H, p.E565D, p.R572C, p.S573F, and p.Y586F) and five ALAS2 truncation mutations (p.P561X, p.V562X, p.H563X, p.E569X and p.F575X) were prokaryotically overexpressed. They were purified to near homogeneity in three steps with a $15-40 \%$ yield; resulting $2-8 \mathrm{mg}$ of enzyme per liter of cells (Table 1; purification data for p.E569X is not shown).

The purification yields of the five missense mutations were all lower than that of the wild-type enzyme (23\%), ranging from a very low $6 \%$ (p.R559H) to $19 \%$ of the initial crude extract activity. After gel filtration chromatography, the ALAS2 missense mutant preparations appeared nearly homogeneous on SDS-PAGE (Fig. 1). The purified wild-type recombinant enzyme existed in two forms of approximately equal amounts by densitometry; $\sim 54$ and $\sim$ $52 \mathrm{kDa}$, with the lower form due to an $\sim 2-\mathrm{kb}$ carboxyl-terminal cleavage that occurred during expression and/or purification, despite the presence of protease inhibitors (Bishop et al. 2013). In addition, all of the missense preparations exhibited, to varying degrees, a third band of approximately $50 \mathrm{kDa}$. This band was prominent in the p.E565D and p.R572C preparations, but was present at much lower quantities in the p.R559H, p.S573F and p.Y586F proteins. It appeared to be a further degradation product of the upper two bands as they diminished in proportion to the appearance of the $\sim 50 \mathrm{kDa}$ band,

In contrast to the case of the missense mutations, the purification of three of the truncated enzymes (p.P561X, p.V562X, p.H563X), resulted in a higher yields (26-46\%) 
Table 1 Purification of recombinant wild-type and mutant ALAS2 ${ }^{\mathrm{a}}$

\begin{tabular}{|c|c|c|c|c|c|}
\hline \multirow[t]{2}{*}{ ALAS2 enzyme } & \multirow[t]{2}{*}{ Step } & Activity & Specific.activity & Yield & Fold Purification \\
\hline & & Units & Units/mg & $\%$ & fold \\
\hline \multirow[t]{3}{*}{ Wild type } & Crude extract & 571,000 & 1130 & 100 & 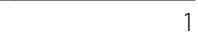 \\
\hline & Affinity chromatography & 323,000 & 21,500 & 56 & 20 \\
\hline & Gel filtration & 131,000 & 93,500 & 23 & 83 \\
\hline \multirow[t]{3}{*}{ p.Arg559His } & Crude Extract & $1,090,000$ & 2090 & 100 & \\
\hline & Affinity chromatography & 358,000 & 29,600 & 33 & 14 \\
\hline & Gel filtration & 95,600 & 108,000 & 8.8 & 52 \\
\hline \multirow[t]{3}{*}{ p.Glu565Asp } & Crude extract & $1,030,000$ & 1710 & 100 & \\
\hline & Affinity chromatography & 825,000 & 50,200 & 80 & 29 \\
\hline & Gel filtration & 194,000 & 122,000 & 19 & 71 \\
\hline \multirow[t]{3}{*}{ p.Arg572Cys } & Crude extract & 267,000 & 780 & 100 & \\
\hline & Affinity chromatography & 143,000 & 8760 & 53 & 11 \\
\hline & Gel filtration & 43,500 & 121,000 & 16 & 155 \\
\hline \multirow[t]{3}{*}{ p.Ser573Phe } & Crude extract & 666,000 & 1070 & 100 & \\
\hline & Affinity chromatography & 301,000 & 11,400 & 45 & 11 \\
\hline & Gel filtration & 84,200 & 115,000 & 13 & 107 \\
\hline \multirow[t]{3}{*}{ p.Tyr586Phe } & Crude Extract & 845,000 & 1650 & 100 & 1 \\
\hline & Affinity chromatography & 287,000 & 15,800 & 34 & 10 \\
\hline & Gel filtration & 128,000 & 109,000 & 15 & 65 \\
\hline \multirow[t]{3}{*}{ P561X } & Crude Extract & $4,660,000$ & 8990 & 100 & 1 \\
\hline & Affinity chromatography & $3,110,000$ & 156,000 & 66 & 17 \\
\hline & Gel Filtration & $1,730,000$ & 288,000 & 37 & 32 \\
\hline \multirow[t]{3}{*}{ V562X } & Crude Extract & $6,410,000$ & 13,300 & 100 & 1 \\
\hline & Affinity chromatography & $2,960,000$ & 116,000 & 46 & $q$ \\
\hline & Gel filtration & $1,330,000$ & 402,000 & 21 & 30 \\
\hline \multirow[t]{3}{*}{ H563X } & Crude Extract & $3,480,000$ & 9070 & 100 & 1 \\
\hline & Affinity chromatography & $2,990,000$ & 170,000 & 86 & 19 \\
\hline & Gel Filtration & $1,610,000$ & 300,000 & 46 & 33 \\
\hline \multirow[t]{3}{*}{ F575X } & Crude Extract & $1,510,000$ & 2990 & 100 & 1 \\
\hline & Affinity chromatography & 355,000 & 30,500 & 23 & 10 \\
\hline & Gel Filtration & 174,000 & 134,000 & 12 & 45 \\
\hline
\end{tabular}

${ }^{\mathrm{a}}$ Data are averages from at least 3 independent purifications

than that of the wild-type enzyme (Table 1). The same result was found in our previous study for the p.Q548X and p.Q581X truncations (Bishop et al. 2013). The ALAS2 truncation mutants p.P561X and pH563X, after fusion-protein cleavage and FPLC purification, migrated to the $52 \mathrm{kDa}$ region (Fig. 2), similar to the p.F557X mutant, which was previously shown to correspond in size to the lower band of wild-type ALAS2 (Ref (Bishop et al. 2013), see Fig. 2). On the other hand, truncation mutant p.V562X migrated further to a position corresponding to a size of around $50 \mathrm{kDa}$. The E569X mutant exhibited both the 50 and $52 \mathrm{kDa}$ bands. The F575X protein migrated to a position slightly lower in size than that of the upper, wild-type $54 \mathrm{kDa}$ bands.
Kinetic and Thermostability properties of the wild-type and mutant ALAS2 enzymes

The specific activities of all the purified missense mutation enzymes were modestly increased from 1.06 to 1.30 times that of the wild-type enzyme. For the substrate succinyl-CoA, the purified mutant enzymes exhibited modest decreases in $\mathrm{K}_{\mathrm{m}}$ (1.1 to 1.5 -fold) consistent with modest increases in $\mathrm{V}_{\max }$, (1.26 to 1.87 fold) (Table 2). The Hill $\mathrm{n}$ numbers were all similar to that of the wild type enzyme. The thermostabilities of the ALAS2 gain-of-function missense mutation enzymes were increased $\sim 2$ - to 3-fold compared to that of the wild-type enzyme. Of interest, this increase was greater, the further the mutation was away from the carboxyl-terminus. 


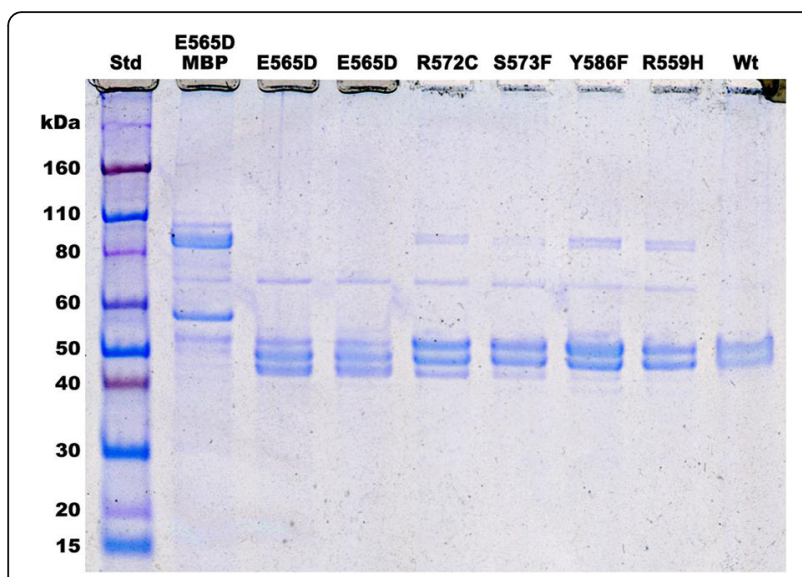

Fig. 1 Protein standards (STD) are Novex Sharp Pre-stained. Lane 2 contains the E565D.MBP fusion protein following affinity purification while all remaining lanes are for enzyme after MBP removal and gel filtration chromatography by FPLC

In contrast to the modest increases in specific activity accompanying missense mutations in the carboxyl-terminal region of ALAS2, truncation mutations in this region markedly increased specific activities from 1.4 to 4.0 fold (Table 3). This was mirrored in similar increases in $\mathrm{V}_{\max }$ for both succinyl-CoA (1.7 to 5.6 fold) and glycine (1.6 to 4.0 fold over wild-type). However, there were only modest changes in the substrate affinities with the $K_{m}$ values for substrate succinyl-CoA being mostly decreased (0.9 to 1.5 fold below wild-type) and the $\mathrm{K}_{\mathrm{m}} \mathrm{s}$ for glycine were mostly increased ( 0.96 to 1.5 -fold over wild-type).

Notably, most of the thermostabilities were significantly reduced with half-lives at $45^{\circ} \mathrm{C}$ of 1.2 to 4.7 -fold decreased over wild-type. For both substrates, therefore, the major kinetic effect was due to greatly increased substrate turnover rather than increases in substrate affinity. Furthermore, unlike the missense mutations, the truncation mutations resulted in enzymes that were more thermolabile than wild-type with half-lives up to 5 times shorter. Interestingly, the most thermolabile mutant had the greatest increases in enzyme rate compared to the wild-type enzyme (Fig. 3).

\section{Discussion}

In our previous publications (Bishop et al. 2012; Bishop et al. 2013) we investigated the biochemical bases of the ALAS2 exon 11 carboxyl-terminal mutations that cause XLP and XLSA. These results demonstrated that some specific mutations could cause loss-of-function and thus XLSA, along with loss of succinyl-CoA synthase binding, while others resulted in gain-of-function by releasing intrinsic inhibition of ALAS2 by its carboxyl-terminus, thus causing XLP. In this study, we examined the kinetic and thermostability properties of additional selected missense and truncation mutations throughout the ALAS2 carboxyl-terminal region in order to further define the structural and kinetic parameters influencing the regulatory properties of ALAS2 exon 11.

The polymorphisms selected for this study constituted all the missense mutations in exon 11 that had been identified by the ESP database. There were five exon 11 mutations in the ESP database. (p.R559H, p.E565D, p.R572C, p.S573F, and p.Y586F) with allele frequencies of 0.009 to $0.294 \%$ (Additional file 2: Table S2). More recently, the spectrum of polymorphisms in this region has been increased and published in the Exome Aggregation Consortium (ExAC) database (Lek et al. 2016). This project achieved a nearly 10 -fold increase in sampling from 6503 individuals (ESP) to 60,706 individuals (ExAC). There were eight additional exon 11 missense mutations found in the ExAC database and the most recent Genome Aggregation Database (gnomAD) (The genome Aggregation Database (gnomAD) 2018) (see Additional file 2: Table S2), but all the additional variants had low allele frequencies of 0.002 to $0.006 \%$, corresponding to 1 to 3 individuals with each mutation. Thus, the survey of the ESP variants represents the vast majority of individuals expected to exhibit possible alteration of function mutations in ALAS2 exon 11.

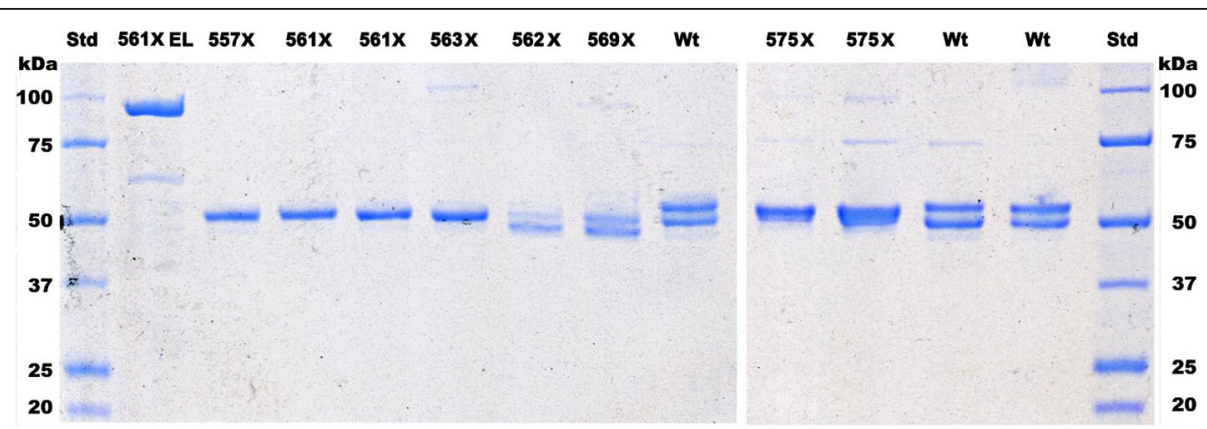

Fig. 2 SDS Page profile of purified ALAS2 truncation (X) mutations. The 561X EL sample is prior to cleavage of the maltose binding protein moiety, while all others are after cleavage. Wt is wild-type ALAS2 and Std are Bio-Rad Precision Plus unstained protein standards. Two different gels were scaled linearly and aligned 
Table 2 Kinetic and thermostability vs predicted properties of ALAS2 missense mutants ${ }^{\mathrm{a}}$

\begin{tabular}{|c|c|c|c|c|c|c|}
\hline Parameters & Wild type & $\mathrm{R} 559 \mathrm{H}$ & E565D & R572C & S573F & Y586F \\
\hline Purified SA (U/mg) & 93,500 & 100,000 & 122,000 & 121,000 & 115,000 & 109,000 \\
\hline Fold-increase & 1.00 & 1.06 & 1. 30 & 1.29 & 1.23 & 1.17 \\
\hline \multicolumn{7}{|l|}{$V_{\max }$} \\
\hline Succinyl-CoA $(U / m g) \pm S t d D e v$ & $93,500 \pm 5300$ & $175,000 \pm 2190$ & $143,000 \pm 7000$ & $136,000 \pm 14,100$ & $120,000 \pm 6710$ & $146,000 \pm 10,100$ \\
\hline Fold-increase & 1.00 & 1.87 & 1.53 & 1.45 & 1.26 & 1.56 \\
\hline \multicolumn{7}{|l|}{$\mathrm{K}_{\mathrm{m}}$} \\
\hline Succinyl-CoA ( $\mu \mathrm{M})$ & $57.1 \pm 6.7$ & $46.3 \pm 6.5$ & $50.8 \pm 2.15$ & $46.7 \pm 1.2$ & $41.1 \pm 9.2$ & $44.0 \pm 5.2$ \\
\hline Fold-decrease & 1.00 & 1.2 & 1.1 & 1.2 & 1.4 & 1.3 \\
\hline Hill coeff. (n) & $1.6 \pm 0.1$ & $1.65 \pm 0.08$ & $1.5 \pm 0.02$ & $1.7 \pm 0.17$ & $1.45 \pm 0.11$ & $1.49 \pm 0.14$ \\
\hline $\mathrm{T}_{1 / 2} 45^{\circ} \mathrm{C}$ (min) & $4.7 \pm 1.4$ & $13.8 \pm 3.4$ & $13.6 \pm 2.8$ & $11.8 \pm 1.5$ & $9.3 \pm 0.8$ & $7.5 \pm 0.2$ \\
\hline \multicolumn{7}{|l|}{ Prediction Program (Ref) and Score ${ }^{b}$} \\
\hline PolyPhen-2 (Adzhubei et al. 2010) & & Benign & Benign & Damaging & Damaging & Benign \\
\hline Score & & 0.003 & 0.001 & 1.000 & 0.995 & 0.016 \\
\hline SIFT (Kumar et al. 2009) & & Tolerated & Tolerated & Deleterious & Deleterious & Tolerated \\
\hline Score & & 0.561 & 0.335 & 0.000 & 0.001 & 0.101 \\
\hline Mutation Assessor (Reva et al. 2011) & & Low & Neutral & Low & Medium & Neutral \\
\hline Score & & 1.32 & -0.285 & 1.94 & 2.12 & 0.46 \\
\hline Provean (Choi et al. 2012) & & Neutral & Neutral & Deleterious & Deleterious & Neutral \\
\hline Score & & -0.45 & -0.47 & -3.28 & -2.75 & -0.89 \\
\hline
\end{tabular}

${ }^{\mathrm{a} D a t a}$ are means $\pm S D$ for $n=4-5$ separate experiments and $\mathrm{V}_{\max }, \mathrm{K}_{\mathrm{m}}$, and $\mathrm{T}_{1 / 2}$ results all show statistically significant differences from the corresponding wild-type values

${ }^{\mathrm{b}}$ Data are the values returned by the online prediction servers for each listed program

The most frequent SNP (p.R559H) was previously identified in an 80 year-old female with late-onset XLSA (Pereira et al. 2004). To date, the present is the only report of this variant's expression, purification and kinetics. The purified mutant enzyme had essentially the same specific activity as the wild-type enzyme.
Kinetically, it had a modestly lower $\mathrm{K}_{\mathrm{m}}$ and higher $\mathrm{V}_{\max }$ for succinyl-CoA (Table 2). However, the purification yield was only $9 \%-60 \%$ lower than that of the wild-type enzyme. This low yield is likely due to proteolysis during purification rather than thermolability since it's half-life at $45^{\circ} \mathrm{C}$ was nearly 3 -fold greater than

Table 3 Kinetic and thermostability properties of ALAS2 truncation mutants ${ }^{a}$

\begin{tabular}{|c|c|c|c|c|c|c|}
\hline Parameters & Wild-Type & P561X & V562X & H563X & E569X & H575X \\
\hline Purified SA (U/mg) & 81,200 & 288,000 & 322,000 & 300,000 & 143,000 & 134,000 \\
\hline Fold-increase & 1.0 & 3.6 & 4.0 & 3.7 & 1.8 & 1.4 \\
\hline$V_{\max }$ Succ. CoA $(U / m g) \pm S D$ & $103,000 \pm 9700$ & $351,000 \pm 25,000$ & $581,000 \pm 88,600$ & $316,000 \pm 3940$ & $182,000 \pm 9570$ & $163,000 \pm 26,600$ \\
\hline Fold-increase & 1.0 & 3.4 & 5.6 & 3.1 & 1.8 & 1.7 \\
\hline$K_{m}$ Succ. CoA $(\mu M) \pm S D$ & $56.1 \pm 6.7$ & $43.3 \pm 2.3$ & $38.5 \pm 2.0$ & $39.6 \pm 4.6$ & $48.8 \pm 5.8$ & $51.2 \pm 2.5$ \\
\hline Fold-decrease & 1.00 & 1.3 & 1.5 & 1.4 & 1.1 & 1.1 \\
\hline Hill coeff. SCoA (n) & $1.6 \pm 0.04$ & $1.5 \pm 0.1$ & $1.6 \pm 0.1$ & $1.6 \pm 0.1$ & $1.8 \pm 0.1$ & $1.6 \pm 0.1$ \\
\hline$V_{\max }$ Gly $(U / m g) \pm S D$ & $96,700 \pm 21,400$ & $324,000 \pm 18,800$ & $381,000 \pm 40,000$ & $316,000 \pm 15,000$ & $156,000 \pm 15,700$ & $153,000 \pm 23,700$ \\
\hline Fold-increase & 1.0 & 3.4 & 4.0 & 3.3 & 1.6 & 1.6 \\
\hline $\mathrm{K}_{\mathrm{m}}$ Gly $(\mathrm{mM}) \pm \mathrm{StdDev}$ & $9.9 \pm 0.5$ & $13.1 \pm 0.7$ & $9.5 \pm 2.2$ & $14.4 \pm 3.1$ & $10.0 \pm 2.0$ & $10.4 \pm 1.3$ \\
\hline Fold-increase & 1.0 & 1.3 & 0.96 & 1.5 & 1.0 & 1.1 \\
\hline $\mathrm{T}_{1 / 2} 45 \mathrm{C}$ (min) \pm StdDev & $4.7 \pm 0.6$ & $2.5 \pm 0.7$ & $1.0 \pm 0.1$ & $3.3 \pm 0.4$ & $3.8 \pm 0.3$ & $N D^{b}$ \\
\hline Fold-decrease & 1.0 & 1.9 & 4.7 & 1.4 & 1.2 & \\
\hline
\end{tabular}

${ }^{a}$ Where provided, data are means \pm SD for $n \geq 3$ separate experiments

${ }^{\mathrm{b}} \mathrm{ND}=$ Not Determined 


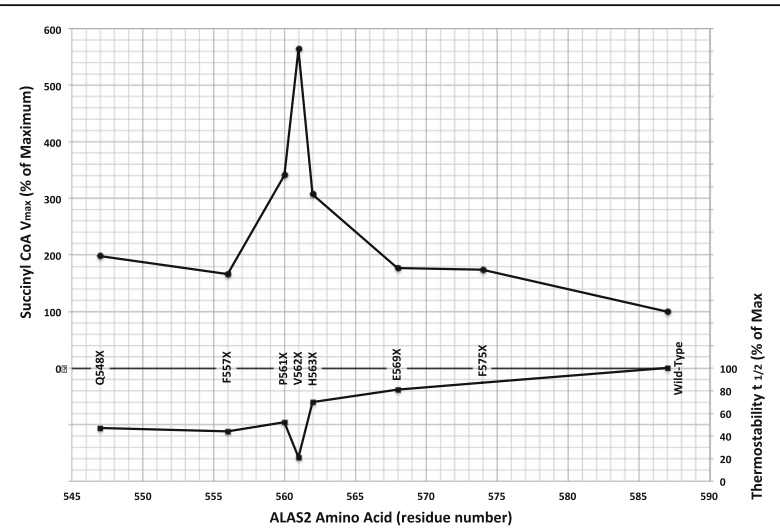

Fig. 3 Effect of Truncation on Succinyl-CoA Vmax and Thermostability. The $V_{\max }$ and thermostability values are from Table 3

that of the wild-type enzyme (Table 2). Since this mild mutation eventually resulted in sideroblastic anemia in an elderly female, these results suggest that the enzyme is more susceptible than wild-type to proteolytic degradation in vivo. Alternatively, it is noted that this mutation is within the region previously suggested to be a site for interaction with succinyl CoA Synthase (Bishop et al. 2012). In those cases, as here, the mutation has little effect on ALAS2 catalytic activity, but results in sideroblastic anemia in vivo (Kadirvel et al. 2012).

To date, there have been no reports of disease associated with the polymorphisms p.E565D, p. R572C or p.S573F. Here, we report that all three SNPs had slightly increased specific activities (1.2- to 1.3-fold), modestly lower $\mathrm{K}_{\mathrm{m}} \mathrm{s}$ and slightly higher $\mathrm{V}_{\max }$ values (1.3- to 1.5 -fold) for succinyl-CoA and 2- to 3-fold greater thermostabilities (Table 2). These properties would suggest modestly increased ALA synthesis in vivo, but possibly not sufficient to cause XLP.

The last missense mutation (p.Y586F) has been previously implicated as an enabler of congenital erythropoietic porphyria (CEP) (To-Figueras, J 2011). In that instance, phenotypic heterogeneity with respect to porphyrin accumulation was discovered in four unrelated CEP patients, all with the same compound heterozygous uroporphyrinogen synthase (UROS) p.C73R/p.P248Q genotype. While three patients had mild disease, one was more severe, with about five-fold higher urinary porphyins than the others. She alone also had an ALAS2 p.Y586F mutation that resulted in about 1.15-fold higher specific activity compared to wild type enzyme. Here, we found that purified p.Y586F enzyme had a $V_{\max }$ for succinyl CoA that was 1.6-fold higher than wild type. Thus, this gain-of-function carboxyl-terminal polymorphism can increase uroporphyrin accumulation in a CEP patient who already had underlying heterozygous UROS mutations (To-Figueras, J 2011).
It was notable that the increase in SNP specific activity was greater, the further the mutation was away from the carboxyl-terminus. Thus, the native conformation of the carboxyl-terminal region may be destabilizing to the enzyme structure, with various point mutations in part relieving this destabilization to a greater extent, the closer the mutation is to residue R559.

The unique role of the ALAS2 carboxyterminal region in the regulation of ALAS2 activity was reflected in the general failure of in silico phenotype prediction programs to correlate with the in vitro expression results reported here. These results are summarized in Table 2 where there is little correlation between predictions and the universal low to modest increase in $\mathrm{V}_{\max }$ for succinyl CoA. This might be expected, since these programs are designed to detect substitutions that have a major deleterious effect locally, where the ALAS2 carboxyl terminal region appears to be more heterogeneous in mechanism with similar amino acid substitutions able to dramatically influence enzyme conformation, typically leading to gain of function, while in other cases, possibly for $\mathrm{R} 559 \mathrm{H}$, to alter an enzyme binding site region (Bishop et al. 2012).

In summary, it appears that the five common SNPs in the ALAS2 carboxyl-terminal region presumably have clinical significance limited to their role as modifying genes - that only in concert with other underlying disturbances of heme biosynthesis may increase ALA synthesis and porphyrin accumulation sufficiently to result in phenotypic disease. These SNPs also did not have a marked effect on ALAS2 kinetics and thus seem more tolerated in the carboxyterminus than mutations elsewhere in the enzyme. Nonetheless, these SNPs should be considered as possible modifier gene mutations that could enhance the symptoms of a porphyria.

In contrast, truncation mutations introduced in this region resulted in high levels of ALAS2 activity typical of mutations causing XLP. This is analogous to most known XLP mutations that cause frameshifts, thereby effectively truncating the ALAS2 C-terminus (Whatley et al. 2008; Bishop et al. 2013). Four of the five new truncation mutations had markedly increased rates of substrate consumption; 1.8 - to 5.6-fold for succinyl-CoA and 1.6- to 4.0-fold for glycine (Table 3).

Similar to results for expressed XLP mutations (Bishop et al. 2013), the p.P561X, p.V562X, and p.H563X truncation mutants showing 1.4- to 4.7-fold reduced half-lives at $45{ }^{\circ} \mathrm{C}$ (Table 3). Of interest, when these data were compared to the succinyl-CoA $\mathrm{V}_{\max }$ values, the basic trend was an inverse relationship (Fig. 3). This could indicate that gain of function in this region is due to increased flexibility or structural "openness" that results in both thermolability and more rapid substrate turnover. In support of this hypothesis, Fratz, et al., have confirmed that the p.Q548X and $\triangle$ AGTG mutations that 
cause XLP have significantly reduced thermostabilities and altered active site structural environments by near-UV circular dichroism spectroscopy, consistent with conformational changes that enhance the rate of succinyl-CoA utilization and product (ALA) release possibly mediated through iron or heme binding to the Cys-X-X-Cys motif located at residues 555 to 558 (Fratz et al. 2015), by interactions with succinyl-CoA synthase near residues 567 and 568 (Bishop et al. 2012) or by the predicted $\alpha$-helical structure of residues 564 to 574 (Bishop et al. 2013). It is therefore important to consider the reciprocal relationship of thermostability and catalytic activity for truncation mutations in the gain-offunction region (Fig. 3). The region of maximal enzyme activity and minimal thermostability coincides with truncations at residues 561 to 563 . Since these truncations are after the Cys-X-X-Cys motif, but before the putative alpha helix, it is more likely that the latter is the key regulatory motif. Thus, the alpha helical region may normally stabilize the protein in a manner that reduces active site conformational flexibility and down-regulates ALA product release.

The variations in subunit sizes of the mutated ALAS2 polypeptides as assessed by denaturing SDS PAGE are of interest. The wild-type enzyme and the SNPs all resulted in two similar forms with apparent molecular weights on SDS gels of around 54 and $52 \mathrm{kDa}$. The upper band corresponds to the full-length peptide from amino acids aspartate 79 to alanine 587 with a calculated molecular weight of $56.4 \mathrm{kDa}$. The lower band, is equivalent to the slightly shorter Asp79 to Asn556 polypeptide (Bishop et al. 2012; Bishop et al. 2013) with a calculated molecular weight of $52.6 \mathrm{KDa}$. The missing 31-amino acid polypeptide has a calculated molecular weight of $3.8 \mathrm{kDa}$. Thus, the apparent molecular weights on SDS gels are about $1-2 \mathrm{kDa}$ less than the actual molecular weights. It should also be noted that mass spectrometry identified Ala88 as the most amino-terminal amino acid (Bishop et al. 2012). This may simply be due to a difficulty in purifying or recovering the first peptide, but if the prokaryotically expressed and purified ALAS2 initiates at Ala88, its predicted $51.6 \mathrm{kDa}$ size is significantly smaller than the observed size in gels.

In contrast to the two molecular sizes observed for the SNPs, the truncation mutations tend to result in only the lower $\sim 52 \mathrm{kDa}$ band. This lower band corresponds to an enzyme form with higher activity than that of the upper band (Table 2, mutant p.F557X, reference \#10), confirming that the native $\mathrm{C}$-terminal residues function as a down-regulator of ALAS2 enzymatic activity. Since the dramatic increase in ALAS2 $V_{\max }$ for succinyl-CoA was not accompanied by a corresponding major increase in substrate affinity (only a modest decrease in $\mathrm{K}_{\mathrm{m}}$ was observed), and since the stabilities of these enzymes were decreased with increasing extent of truncation, it remains that the increased turnover is most likely due to enhanced product release due to the loss of the C-terminal residues.

The presence of the putative alpha helix at residues 564-574. (Bishop et al. 2013) appears to be important, as we show that the maximum gain-of-function occurred for truncations of any of the three residues just prior to the alpha helix region. It appears that contact of the helix with the ALAS2 protein may increase its rigidity and/or compactness, protecting it from proteolysis and reducing its conformational flexibility. Conversely, when truncated, the increased flexibility may result in easier product release, and the consequent major increase in activity. Previously, Lendrihas, Hunter and Ferreira (Lendrihas et al. 2010; Hunter and Ferreira 2011) showed that conformational flexibility was critical for ALA release from ALAS2. In their study, mutations in an active site-resident loop in the murine enzyme corresponding to human exon 10 residues 500 to 517 were proposed to alter ALA release rate via conformational change of the loop. The shortened half-lives for thermal denaturation of the ALAS2 truncation mutations was consistent with an increase in instability and flexibility of the protein that could result in the observed increased in enzymatic activity due to increased rates of ALA release. Stojanovski, et al., has also recently suggested that truncation mutations that change the ALAS2 tertiary structure from a closed to open conformation may increase activity by releasing product more rapidly and may decrease thermostability by reducing the rigidity of the structure (Stojanovski et al. 2016).

Of note, while this manuscript was in review, the structure of erythroid-specific ALAS2 (PDB ID $6 \mathrm{HRH}$ ) was deposited in the RCSB PDB Database (Bailey et al. 2018). Viewing the deposited structure with the MacPyMol molecular visualizer (The PyMOL Molecular Graphics System 2018) shows that the region around F557 is unstructured and does not appear in the deposited crystal structure. This supports our findings that mutations and deletions in the carboxyl terminus distal to this region could result in the enzyme being more susceptible to protease activity. Most importantly, our viewing the stereo image of the deposited structure reveals that the above-described alpha helical structure consisting of residues S566 to F573, lies on the surface of each subunit directly above the active site loop reported by Lendrihas, (Lendrihas et al. 2010) as well as above the PLP active site moiety. Thus it is clear that this helix could inhibit ALA release as well as promoting enzyme rigidity, thus providing a rationale as to why the maximum deletion mutant activation centers on this helix (see Fig. 3). 
The regulatory nature of the ALAS2 carboxyl-terminus has been shown here to significantly involve an alpha helix near the center of a eukaryotic-specific sequence extension of the core ALAS sequence found in prokaryotes. The mitochondrial foldase-protease complex, ClpXP has also been shown to play a significant role in ALAS2 regulation in that ClpX unfoldase activates ALAS2 by stimulating incorporation of cofactor pyridoxal 5 '-phosphate (PLP) into ALAS2 (Kardon et al. 2015) and that a mutation in ClpX modulates ALAS2 levels in humans (Yien et al. 2017). Since the new crystal structure of ALAS2 shows that there is a cone-shaped access hole to PLP that is near the carboxyl-terminus, it would be interesting to know if this region is involved with ClpXP. Importantly, Kubota, et al. showed that the Heme Regulatory Motif, amino acids CPF/L (Lathrop and Timko 1993), present in mitochondrial ALAS2 mediated heme-dependent recruitment of ClpPX for degradation of ALAS2 (Kubota et al. 2016). Since there is a surface-localized CPL sequence at the cone-shaped entrance to the PLP binding site near the carboxyterminus, the involvement of the eukaryotic-specific carboxyterminus extension may be of importance to multiple aspects of the regulation and control of ALAS2 activity.

\section{Conclusions}

An ALAS2 C-terminal SNP (p.R559H) had markedly low purification yield suggesting enzyme proteolytic instability as a possible cause for the previously identified late-onset patient with XLSA.

Four relatively common SNPs (p.E565D, p.R572C, p.S573F, and p.Y586F) in the exon $11 \mathrm{C}$-terminal region were associated with small increases in enzymatic activity in vitro, and are likely biochemical and clinical modifiers of porphyria severity.

Synthetic ALAS2 exon 11 truncation mutations centered on an active-site-covering alpha helix resulted in dramatic increases in succinyl-CoA turnover that were largely independent of changes in substrate affinity, thus correlating increased thermolability and presumably increased conformational flexibility with enhanced ALA product release.

\section{Additional files}

Additional file 1: Table S1. Oligonucleotides primers ( 5 ' to 3 'forward) for mutagenesis and sequencing of ALAS2. (DOC $36 \mathrm{~kb}$ )

Additional file 2: Table S2. Allele frequencies of ALAS2 exon 11 SNPs in the ESP, EXAC and GnomAD databases. (DOC $25 \mathrm{~kb}$ )

\section{Abbreviations}

ALA: 5-Aminolevulinic acid; ALAS2: 5-Aminolevulinic acid synthase, erythroid isozyme; CEP: Congenital erythropoietic porphyria; CoA: Coenzyme A; ESP: Exome Sequencing Project; ExAC: Exome Aggregation Consortium; $\mathrm{K}_{\mathrm{m}}$ : Michaelis kinetic constant; SDS-PAGE: Sodium dodecyl sulfate - polyacrylamide gel electrophoresis; SNP: Single nucleotide polymorphism; UROS: Uroporphyrinogen synthase; $V_{\text {max }}$ : Maximum reaction velocity at saturating substrate; XLP: X-linked protoporphyria; XLSA: X-linked sideroblastic anemia

\section{Acknowledgements}

The authors thank Wanqiong Qido for technical assistance and Senkottuvelan Kadirvel for generation and purification of an expression construct (E569X) used in these studies.

\section{Funding}

This research was supported in part by a grant (5 R01 DK26824) from the National Institutes of Health $(\mathrm{NIH})$, royalties form an invention in another field, and funds from the Department of Genetics and Genomic Sciences, Icahn School of Medicine at Mount Sinai.

\section{Availability of data and materials}

The authors declare that all data supporting the findings of this study are available within the article and its supplementary information files.

\section{Authors' contributions}

$\mathrm{VT}$ did the experimental work and wrote a first draft of the MS. RD conceived of the study and provided an edit of the final manuscript. DB designed all experiments, provided technical support, interpreted the results, and wrote and edited the manuscript. All authors read and approved the final manuscript.

Ethics approval and consent to participate

Not applicable.

\section{Consent for publication}

Not applicable.

\section{Competing interests}

The authors declare that they have no competing interests.

\section{Publisher's Note}

Springer Nature remains neutral with regard to jurisdictional claims in published maps and institutional affiliations.

Received: 31 August 2018 Accepted: 2 January 2019

Published online: 24 January 2019

\section{References}

Adzhubei IA, Schmidt S, Peshkin L, Ramensky VE, Gerasimova A, Bork P, Kondrashov AS, Sunyaev SR. A method and server for predicting damaging missense mutations. Nat Methods. 2010;7:248-9.

Astner I, et al. Crystal structure of 5-aminolevulinate synthase, the first enzyme of heme biosynthesis and its link to XLSA in human. EMBO J. 2005;24:3166-77.

Bailey, HJ, Shrestha, L, Rembeza, E, Newman, J, Kupinska, K, Diaz-Saez, L, Kennedy, E, Burgess-Brown, N, von Delft, F, Arrowsmith, C, Edwards, A, Bountra, C, Yue, WW, Structural Genomics Consortium (SGC). Structure of human erythroidspecific 5'-aminolevulinate synthase, ALAS2 (www.rcsb.org/structure/6HRH ). [Cited Dec 6, 2018].

Bishop DF, Henderson AS, Astrin KH. Human $\delta$-aminolevulinate synthase: assignment of the housekeeping gene to 3 p21 and the erythroid-specific gene to the X-chromosome. Genomics. 1990;7:207-14.

Bishop DF, Tchaikovskii V, Hoffbrand AV, Fraser ME, Margolis S. X-linked sideroblastic anemia due to carboxyl-terminal ALAS2 mutations that cause loss of binding to the $\beta$-subunit of succinyl CoA synthase (SUCLA2). J Biol Chem. 2012;287:28943-55.

Bishop DF, Tchaikovskii V, Nazarenko I, Desnick RJ. Molecular expression and characterization of erythroid-specific 5-aminolevulinate synthase gain-offunction mutations causing X-linked protoporphyria. Mol Med. 2013;19:18-25.

Brown BL, Kardon JR, Sauer RT, Baker TA. Structure of the mitochondrial aminolevulinic acid synthase, a key heme biosynthetic enzyme. Structure. 2018;26:1-10.

Choi Y, Sims GE, Murphy S, Miller JR, Chan AP. Predicting the functional effect of amino acid substitutions and Indels. PLoS One. 2012;7:e46688. 
Cotter PD, Baumann M, Bishop DF. Enzymatic defect in X-linked sideroblastic anemia: molecular evidence for erythroid $\delta$-aminolevulinate synthase deficiency. Proc Natl Acad Sci U S A. 1992;89:4028-32.

Ducamp $\mathrm{S}$, et al. Molecular and functional analysis of the C-terminal region of human erythroid-specific 5-aminolevulinic synthase associated with X-linked dominant protoporphyria (XLDPP). Hum Mol Genet. 2013;22:1280-8.

Exome Variant Server, NHLBI GO Exome Sequencing Project (ESP), Seattle, WA (http://evs.gs.washington.edu/EVS/) [cited Aug 28, 2017].

Fratz $\mathrm{E}$, et al. Human erythroid 5-aminolevulinate synthase mutations associated with X-linked protoporphyria disrupt the conformational equilibrium and enhance product release. Biochemistry. 2015:54:5617-31.

Hunter GA, Ferreira GC. Molecular enzymology of 5-aminolevulinate synthase, the gatekeeper of heme biosynthesis. Biochim Biophys Acta. 2011;1814:1467-73.

Kadirvel S, et al. The carboxyl-terminal region of erythroid-specific 5aminolevulinate synthase acts as an intrinsic modifier for its catalytic activity and protein stability. Exp Hematol. 2012;40:477-86.

Kardon JR, et al. Mitochondrial ClpX activates a key enzyme for heme biosynthesis and erythropoiesis. Cell. 2015;161:858-67.

Kubota Y, et al. Novel mechanisms for heme-dependent degradation of ALAS1 protein as a component of negative feedback regulation of heme biosynthesis. J Biol Chem. 2016;291:20516-29.

Kumar P, Henikoff S, Ng PC. Predicting the effects of coding non-synonymous variants on protein function using the SIFT algorithm. Nat Protoc. 2009;4:1073-81.

Lathrop JT, Timko MP. Regulation by heme of mitochondrial protein transport through a conserved amino acid motif. Science. 1993;259:522-5.

Lek $\mathrm{M}$, et al. Analysis of protein-coding genetic variation in 60,706 humans. Nature. 2016;536:285-91.

Lendrihas T, Hunter GA, Ferreira GC. Targeting the active site gate to yield hyperactive variants of 5-aminolevulinate synthase. J Biol Chem. 2010;285: 13704-11.

Pereira JC, Gonçalves P, Cunha E, Ribeiro ML. Gene symbol: ALAS2, disease: Sideroblastic Anemia. Hum Genet. 2004;115:533.

Reva B, Antipin Y, Sander C. Predicting the functional impact of protein mutations: application to cancer genomics. Nucl Acids Res. 2011;39:e118.

Stenson PD, et al. The human gene mutation database: towards a comprehensive repository of inherited mutation data for medical research, genetic diagnosis and next-generation sequencing studies. Hum Genet 2017;136:665-77.

Stojanovski BM, Breydo L, Uversky VN, Ferreira GC. Murine erythroid 5aminolevulinate synthase: truncation of a disordered $\mathrm{N}$-terminal extension is not detrimental for catalysis. Biochim Biophys Acta. 2016;1864:441-52.

The genome Aggregation Database (gnomAD) ( https://macarthurlab.org/2017/ 02/27/the-genome-aggregation-database-gnomad /) [cited Apr 23, 2018].

The PyMOL Molecular Graphics System, Version 2.0 Schrödinger, LLC. ( www. PyMol.org ). [cited Dec 6, 2018].

To-Figueras, J, et al. ALAS2 acts as a modifier gene in patients with congenital erythropoietic porphyria. Blood. 2011;118:1443-51.

Whatley SD, et al. C-terminal deletions in the ALAS2 gene lead to gain of function and cause X-linked dominant protoporphyria without anemia or iron overload. Am J Hum Genet. 2008:83:408-14.

Yien YY, et al. Mutation in human CLPX elevates levels of delta-aminolevulinate synthase and protoporphyrin IX to promote erythropoietic protoporphyria. Proc Nat Acad Sci. 2017;114:e8045-52.

Ready to submit your research? Choose BMC and benefit from:

- fast, convenient online submission

- thorough peer review by experienced researchers in your field

- rapid publication on acceptance

- support for research data, including large and complex data types

- gold Open Access which fosters wider collaboration and increased citations

- maximum visibility for your research: over $100 \mathrm{M}$ website views per year

At $\mathrm{BMC}$, research is always in progress.

Learn more biomedcentral.com/submissions 